\title{
Convective chemical-wave propagation in the Belousov-Zhabotinsky reaction
}

\author{
Yunqing Wu, ${ }^{1}$ Desiderio A. Vasquez, ${ }^{2}$ Boyd F. Edwards, ${ }^{1}$ and Joseph W. Wilder ${ }^{3}$ \\ ${ }^{1}$ Department of Physics, West Virginia University, P.O. Box 6315, Morgantown, West Virginia 26506-6315 \\ ${ }^{2}$ Department of Physics, Indiana University-Purdue University at Fort Wayne, Fort Wayne, Indiana 46805-4199 \\ ${ }^{3}$ Department of Mathematics, West Virginia University, P.O. Box 6310, Morgantown, West Virginia 26506-6310
}

(Received 17 August 1994)

\begin{abstract}
We investigate the onset of convection for chemical-wave propagation in the Belousov-Zhabotinsky reaction based on the two-variable Oregonator model coupled with the fluid dynamic equations. For chemical waves in a vertical slab, two-dimensional convection occurs only for slab widths greater than a critical threshold width. The convective threshold is different for ascending and descending waves. Convectionless waves are flat and propagate with constant speed. Above the onset of convection, the wave velocity increases and the flat wave deforms due to two counterrotating steady rolls. For a horizontal slab, convection is always present and the wave velocity increases with increasing slab width. Our results are compared with experiments.
\end{abstract}

PACS number(s): 47.20.Bp, 47.70.Fw, 82.20.Mj

\section{INTRODUCTION}

In the past two decades, chemical oscillations and pattern formation in nonequilibrium chemical systems have been interesting and fruitful research areas [1]. Many phenomena, such as reaction-front propagation, target patterns, spiral waves, and periodic wave trains, can be described by reaction-diffusion mechanisms. Nevertheless, as most experiments are performed in aqueous solutions, convection can play a role in these processes since different chemical compositions induce density gradients [2]. Experiments by McManus et al. and Masere et al. [3] in the iodate-arsenous acid system have shown that upward propagating fronts are curved and their velocities are enhanced for large tube diameters $(\geq 1.1 \mathrm{~mm})$, while for small tube diameters $(<0.94 \mathrm{~mm})$ and downward propagating waves, the front is flat and propagates with constant speed independent of the tube diameter $[2,3]$. For tube diameters up to $1.6 \mathrm{~mm}$, they observed nonaxisymmetric curved wave fronts. If the diameter exceeded $2.3 \mathrm{~mm}$, a completely axisymmetric wave front was observed. For the strongly exothermic iron (II)-nitric acid system, Epstein and co-workers [4] observed that the speed of the front was sensitive to the width of the tube and to the orientation with respect to gravity. Ascending fronts propagate faster than pure reaction-diffusion fronts and descending fronts propagate with velocities up to 80 times the velocity of pure reaction-diffusion fronts. Nagypal, Bazsa, and Epstein [5] have shown that the front speed in the chlorite-thiosulfate reaction depends on the direction of propagation with respect to the gravitational field. A series of delicate experiments in petri dishes by Miike and co-workers [6] has established that convective rolls are associated with chemical waves in the BelousovZhabotinsky (BZ) reaction. They also observed oscillatory hydrodynamic flow and periodic deformation of wave fronts induced by spiral wave propagation [6]. A recent experiment by Menzinger et al. [7] in a vertical tube has further demonstrated the onset of convection and the oc- currence of turbulence induced by upward chemical wave propagation. In actual experiments, many external factors may affect the onset of convection, such as surface tension, surface evaporative cooling, or even inhomogeneities in the reagents. Nevertheless, the experiments by Miike and co-workers [6] strongly indicate that hydrodynamic flow can exist in the absence of these external effects, being induced exclusively by chemical waves in the $\mathrm{BZ}$ reaction. In this paper, we consider the convective flows resulting from chemical wave propagation in the $\mathrm{BZ}$ reaction.

Chemical waves may induce a density gradient within the wave profile either by changes in chemical composition or by changes in temperature owing to reaction exothermicity [2]. According to the estimate of Pojman and Epstein [2], the BZ reaction is not highly exothermic; its small increase in temperature across the front is only about $0.1^{\circ} \mathrm{C}$. In fact, since the typical thermal diffusivities $\left(\approx 10^{-3} \mathrm{~cm}^{2} / \mathrm{s}\right)$ are about 100 times greater than typical molecular diffusivities $\left(\approx 10^{-5} \mathrm{~cm}^{2} / \mathrm{s}\right)$, any thermal gradient can be rapidly smoothed out by thermal diffusion, leaving the density gradient practically the same as the purely compositional density gradient at the higher temperature of the reacted fluid. Previous calculations on the iodate-arsenous acid reaction have justified this argument [8]. Therefore, in this paper, we consider only the density gradient induced by changes in chemical composition. Even though a complete description of the experiments in cylinders and petri dishes requires the use of three-dimensional geometry, we will confine our attention to the simpler two-dimensional slab geometry. The problem in the cylinder is much more complicated because of its three-dimensional nature and the singularity at the origin in cylindrical coordinates. Our previous calculations for the iodate-arsenous acid reaction in a twodimensional slab [9] predicted a critical width for the onset of convection that agrees with experiments in a cylinder. The experimental measurements in petri dishes using two-dimensional spectrophotometery [6] show that 
the lateral flow velocity induced by a target pattern is negligible compared with the strong flow velocity in the direction of the chemical-wave propagation, further suggesting that the slab geometry is a good approximation.

Based on this simplified model, we investigate the onset of convection induced by chemical-wave propagation in the $\mathrm{BZ}$ reaction for both vertical and horizontal slabs. For vertical slabs, we find critical slab widths for both ascending and descending waves. If the slab width is greater than the critical width, the wave curves and the chemical-wave velocity increases due to two counterrotating rolls. For the horizontal slab, convection is always present and the wave velocity increases monotonically with the slab width. Two different wave front shapes represent two possible density profiles in the $\mathbf{B Z}$ reaction. The present work is, as far as we know, the first nonlinear theoretical treatment for convective wave propagation in the BZ reaction. Our results are compared with experiments.

\section{EQUATIONS OF MOTION}

To describe the reaction-diffusion waves in the $\mathrm{BZ}$ reaction, we use the two-variable Oregonator model coupled with diffusion. This model provides a good description of oscillations, bistability, expanding target patterns, and spiral waves in the $\mathbf{B Z}$ reaction $[1,10,11]$ :

$$
\begin{aligned}
& \frac{\partial u}{\partial t}=D \nabla^{2} u+\frac{1}{\varepsilon}\left(u-u^{2}-f v \frac{u-q}{u+q}\right), \\
& \frac{\partial v}{\partial t}=D \nabla^{2} v+u-v .
\end{aligned}
$$

Here, $u$ and $v$ respectively represent the dimensionless concentrations $\left[\mathrm{HBrO}_{2}\right]$ and $\left[\mathrm{Ce}^{4+}\right]$, and $f, q, \varepsilon$, and $D$ are constants related to the chemical kinetics and initial chemical concentrations [10,11]. For a typical recipe used to investigate waves in excitable $\mathrm{BZ}$ reagent, we have a scaling with a spatial unit of $0.018 \mathrm{~cm}$ and a time unit of $21 \mathrm{sec}$ [11] using the "Lo" kinetic values cataloged by Keener and Tyson [10]. Under this scaling, those constants are $f=3, \varepsilon=0.01, q=2 \times 10^{-4}$, and $D=1$ [11]. Here we have used the dimensional molecular diffusivity $D_{c}=1.5 \times 10^{-5} \mathrm{~cm}^{2} / \mathrm{sec}$. This scaling was first introduced by Jahnke, Skaggs and Winfree [11] to simulate the vortex dynamics of spiral waves, which makes the diffusivity equal to 1 instead of $\varepsilon$. Equations (1) and (2) have a stable steady state $\left(u_{s}, v_{s}\right)$ with $u_{s}=v_{s}=\left[\sqrt{(q+f-1)^{2}+4 q(1+f)}-(q+f-1)\right] / 2$. For our choice of parameters and dimensionless units, $u_{s}=3.9984 \times 10^{-4}$. It has been shown that this system is excitable if $f>1+\sqrt{2}$. This means that a strong enough localized perturbation around the steady state will eventually develop into a propagating pulse as shown in Fig. 1. Inside the pulse, $u$ first deviates from the steady state quickly (fast variable), which triggers the initial increase of $v$ (slow variable). After $u$ reaches its maximum value $(\approx 0.856)$, it gradually recovers to the steady state. It should be pointed out that both $u$ and $v$ have extended recovery tails, even though $u$ 's tail is indistinguishable from the $u=0$ line since it is so close to the steady state.

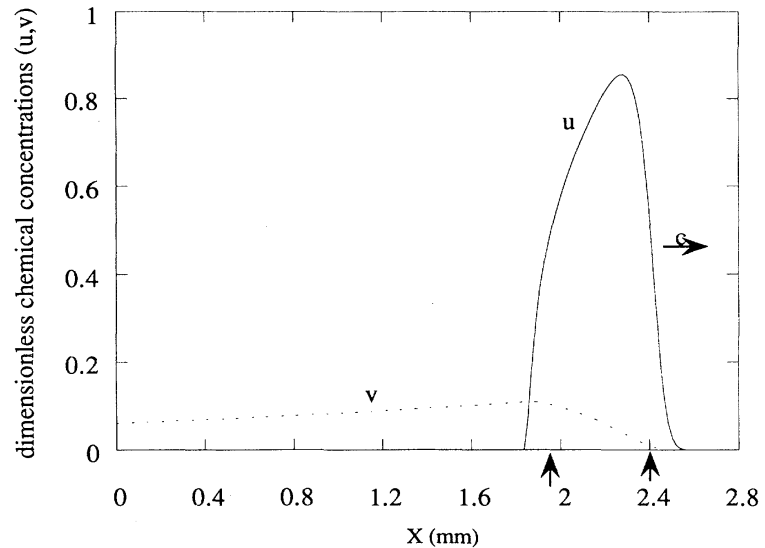

FIG. 1. Pulse profiles of traveling wave solutions to Eqs. (1) and (2). The pulse travels with a constant speed of $C=17.41$ in dimensionless unit $(8.95 \mathrm{~mm} / \mathrm{min})$. The arrows indicate the locations of the front and the tail. The solid and dashed lines represent the variables $u$ and $v$, respectively.

For later reference, we define the position at which $u$ goes above $u_{\max } / 2=0.428$ as the front position and the position at which $u$ goes below 0.428 as the tail position, as indicated by arrows in Fig. 1. This pulse propagates with constant dimensionless speed of 17.41 , or $8.95 \mathrm{~mm} / \mathrm{min}$ in conventional units.

To account for the convection of chemical species, we have to couple the hydrodynamic equations of motion with the above reaction-diffusion equations, which leads to the following set of equations:

$$
\begin{aligned}
& \frac{\partial \mathbf{V}}{\partial t}+(\mathbf{V} \cdot \boldsymbol{\nabla}) \mathbf{V}=\frac{\rho-\rho_{0}}{\rho_{0}} \mathbf{g}-\frac{1}{\rho_{0}} \nabla P+v \nabla^{2} \mathbf{V}, \\
& \boldsymbol{\nabla} \cdot \mathbf{V}=0, \\
& \frac{\partial u}{\partial t}+\mathbf{V} \cdot \nabla u=D \nabla^{2} u+\frac{1}{\varepsilon}\left[u-u^{2}-f v \frac{u-q}{u+q}\right), \\
& \frac{\partial v}{\partial t}+\mathbf{V} \cdot \nabla v=D \nabla^{2} v+u-v .
\end{aligned}
$$

Here, $\mathbf{V}$ is the fluid velocity, $P$ is the reduced pressure and is related to the conventional pressure by $P=p-\rho_{0}\left(g_{x} x+g_{z} z\right), \mathbf{g}=\left(g_{x}, g_{z}\right)$ is the acceleration of gravity, $v$ is the kinematic viscosity, and $D$ is the molecular diffusivity. By writing equations in this form, all hydrodynamic variables are expressed in terms of the scaling of Eqs. (1) and (2). That is, $g=980 \mathrm{~cm} \mathrm{sec}^{-2} \times(21$ $\mathrm{sec})^{2} /(0.018 \mathrm{~cm})=2.4 \times 10^{7}, \quad v=9.2 \times 10^{-3} \mathrm{~cm}^{2} \mathrm{sec}^{-1}$ $\times(21 \mathrm{sec}) /(0.018 \mathrm{~cm})^{2}=5.96 \times 10^{2}$. To complete our equations, we need another relation between density and chemical concentration. For most known reactions, the density variation due to the change in chemical composition is quite small, being typically of order $10^{-4}$. We can therefore assume a linear dependence of density on the chemical concentrations 


$$
\rho=\rho_{0}\left[1-\beta_{u}\left(u-u_{s}\right)-\beta_{v}\left(v-v_{s}\right)\right] .
$$

Here, $\rho_{0}$ is the density of the bulk fluid, and $\beta_{u}$ and $\beta_{v}$ are the coefficients of linear expansion due to the compositional change of $u$ and $v$. Pojman and Epstein [2] suggest that the density change may occur mostly during the catalyst oxidation process, so the density should mainly depend on $v$. But the long tail of $v$ requires a very large mesh size to simulate single-pulse propagation. Consequently, a much longer computer time is required for each iteration. Furthermore, since the Oregonator is a simplified model of the BZ reaction, it may not precisely account for the chemical dependence of the fluid variables. Since there exist no definitive experimental results about the relative importance of $\beta_{u}$ and $\beta_{v}$, we here assume that the density only depends on $u$ for this first calculation [12]. This simplification will still show the effects of a pulselike density profile. As $u$ 's profile is much more confined than $v$ 's profile, we can use smaller spatial domain and reduce our computational time. In this case, Eq. (7) simplifies to

$$
\rho=\rho_{0}\left[1-\beta\left(u-u_{s}\right)\right] \text {. }
$$

If $\beta>0$ in Eq. (8), the reacting region is less dense than the remaining fluid. Existing experimental data indicate that the sign of $\beta$ depends on the catalysts used in the BZ reaction [13]. In a vertical-tube experiment for $\mathrm{Mn}^{2+}$. catalyzed $\mathrm{BZ}$ reaction, Menzinger et al. [7] observed the onset of convection and acceleration of chemical waves for upward propagation; while for downward propagation, they observed steady propagation without convection, thus concluding that the reacting region is lighter than the steady fluid $(\beta>0)$ [7]. In contrast, an estimate made for $\mathrm{Ce}^{2+}$ - and $\mathrm{Fe}^{2+}$-catalyzed $\mathrm{BZ}$ reaction by Pojman and Epstein [2] indicates that the reacting fluid should be heavier than the remaining fluid $(\beta<0)$. We will first study the case where the reacting fluid is lighter $(\beta>0)$, and later the opposite case $(\beta<0)$.

The magnitude of $\beta$ can be obtained from experimental measurements of the isothermal fractional density difference $\delta=\left(\rho_{0}-\rho\right) / \rho_{0}$ for bulk oscillating fluid using $\beta=\delta /\left(u_{\max }-u_{s}\right)$. Here $u_{\max }-u_{s} \approx 0.856$. The only experimental measurement [7] for $\delta$ in the $\mathrm{Mn}^{2+}$-catalyzed $\mathrm{BZ}$ reaction gives the extremely small value $\delta \approx 10^{-7}$. Another estimate [2] based on the molar volume change in the $\mathrm{Fe}^{2+}$-catalyzed $\mathrm{BZ}$ reaction gives $\delta=2 \times 10^{-5}$. For other chemical waves, namely the iodate-arsenous acid reaction and the iron-nitric acid reaction, $\delta=0.87 \times 10^{-4}$ and $5.5 \times 10^{-4}$, respectively $[3,4,8]$. Thus, estimates of $\beta$ vary widely for different reactions and different catalysts used. In the present calculation, we take $\beta=2.0 \times 10^{-3}$. Compared to the above data, this value is somewhat large. We choose this value because the two variable Oregonator is a simplified model of the $\mathrm{BZ}$ reaction, so an exact value for $\beta$ will not lift the uncertainty introduced by the reaction-diffusion model. The choice of a somewhat large $\beta$ makes our calculations computationally more efficient and are expected to give the correct qualitative behavior. With these simplifications, our results should be considered as qualitative ones for this very first convective model of a pulse- like chemical wave.

We can eliminate the reduced pressure and satisfy continuity identically by defining the stream function $\psi$ as

$$
V_{x}=\frac{\partial \psi}{\partial z}, \quad V_{z}=-\frac{\partial \psi}{\partial x},
$$

and the vorticity $\omega$ as

$$
\omega=\nabla^{2} \psi \text {. }
$$

Combining with Eq. (8), we can rewrite the equations as

$$
\begin{aligned}
& \frac{\partial \omega}{\partial t}=\frac{\partial(\psi, \omega)}{\partial(x, z)}+\beta g_{z} \frac{\partial u}{\partial x}-\beta g_{x} \frac{\partial u}{\partial z}+v \nabla^{2} \omega, \\
& \frac{\partial u}{\partial t}=\frac{\partial(\psi, u)}{\partial(x, z)}+D \nabla^{2} u+\frac{1}{\varepsilon}\left[u-u^{2}-f v \frac{u-q}{u+q}\right), \\
& \frac{\partial v}{\partial t}=\frac{\partial(\psi, v)}{\partial(x, z)}+D \nabla^{2} v+u-v,
\end{aligned}
$$

where we have used the Jacobi operator

$$
\frac{\partial\left(f_{1}, f_{2}\right)}{\partial(x, z)}=\frac{\partial f_{1}}{\partial x} \frac{\partial f_{2}}{\partial z}-\frac{\partial f_{2}}{\partial x} \frac{\partial f_{1}}{\partial z} \text {. }
$$

Equations (10), (11), (12), and (13) completely define our problem. The boundary conditions are no-slip boundary conditions for the fluid velocity and no-flow boundary conditions for the chemical concentrations at the slab walls. We also require zero fluid velocity far ahead and far behind the pulse, where there is no density gradient. For the chemical concentrations, we use the fixed steady state far ahead of the pulse and the equal slope boundary conditions far behind the pulse to allow for the slow recovery of $u$ and $v$.

\section{NUMERICAL METHOD}

The system of Eqs. (10), (11), (12), and (13) was solved numerically. Our previous computations on the iodatearsenous acid system indicate that relaxation of a finitedifference form of Eq. (10) takes many iterations for each time step [9]. Instead we use a simpler and equally valid finite-term expansion for the stream function [14]. For the two-dimensional slab, the stream function can be expanded in a series of complete orthonormal eigenfunctions of the operator $d^{4} / d x^{4}$,

$$
\frac{d^{4}}{d x^{4}} \mathcal{T}_{m}=\lambda_{m}^{4} \mathcal{T}_{m}
$$

satisfying $\mathcal{T}_{m}=d \mathcal{T}_{m} / d x=0$ at $x= \pm \frac{1}{2}$. These functions are divided into two classes: $C_{m}(x)$ for even functions and $S_{m}(x)$ for odd functions. The functions and their eigenvalues $\lambda_{m}$ are tabulated by Chandrasekar [15]. Accordingly the stream function becomes

$$
\psi(x, z, t)=\sum_{m=1}^{\infty} A_{m}(z, t) \mathcal{T}_{m}(x / d),
$$

where $d$ is the wall separation. Truncating the series will provide a good approximation for flows near the onset of convection. This expansion converges quite rapidly for the linear stability analysis of chemical waves in the 
iodate-arsenous acid reaction [14]. In this way, the twodimensional stream function is effectively reduced to a one-dimensional problem, thus greatly simplifying the work. Details of this method are given in Ref. [9]. For our problem, we have used a one-term truncation with $C_{1}(x)$ as well as a four-term truncation including $C_{1}(x)$, $C_{2}(x), S_{1}(x)$, and $S_{2}(x)$. The one-term truncation is valid only when the system is very close to the onset of convection. The coefficients $A_{m}(z, t)$ become larger and the complexity of the convective flow increases as the system is taken further above the onset of convection. A fourterm truncation provides a good check on the validity of the one-term truncation as well as the convergence of our expansion. In a typical calculation in a vertical slab with $d=0.4 \mathrm{~mm}$, maximum absolute values of $A_{m}(z, t)$ are $A_{c_{1}}=0.379, A_{c_{2}}=0.010, A_{s_{1}}=0.022$, and $A_{s_{2}}=0.001$. It is clear in this case that the convective flow mainly contains the contributions of $C_{1}(x)$ and that the contributions of $S_{1}(x), C_{2}(x)$, and $S_{2}(x)$ are much smaller.

We test our numerical results in two ways. First, we study an eight-term truncation including $C_{1}(x)$ to $C_{4}(x)$ and $S_{1}(x)$ to $S_{4}(x)$ to verify that the terms ignored in the four-term truncation are negligible. Second, we use the subroutine GENBUN from the FISHPAK package for solving elliptic partial differential equations [16]. Both tests yield chemical speeds that differ from the four-term truncation by less than $0.3 \%$. One advantage of our algorithm is that it runs three times faster than the GENBUN subroutine.

It is known that the Oregonator model has stiff kinetics because of the smallness of $\varepsilon$. The explicit Euler method for this system requires a very small time step. Switching to an implicit method allows a time step about 20 times larger. However, the large value of $v$ in Eq. (11) prevents us from using this larger time step when coupled with convection. Consequently, we simply use an explicit Euler method. We define $z$ as the direction of wave propagation and $x$ as the direction perpendicular to $z$. The chemical concentrations and fluid velocities fall off approximately exponentially well ahead of the front $(z \rightarrow \infty)$ and well behind the tail $(z \rightarrow-\infty)$. To determine acceptable cutoffs ahead of the front and behind the tail to facilitate numerical computations on a finite domain, we fix the mesh-point separations $\Delta x=0.2$ and $\Delta z=0.1$, but vary the number of mesh points in the $z$ direction. For a vertical slab with $d=0.5 \mathrm{~mm}$, we find that the mesh sizes $400 \times 15,300 \times 15$, and $200 \times 15$ give the same results. We therefore use the $200 \times 15$ mesh. To test the validity of using 15 mesh points in the $x$ direction for the same $d$, we increase the mesh size to $200 \times 30$, while using $\Delta x=0.1$. We find that this procedure changes the chemical wave speed by less than $0.4 \%$. For a given spatial interval, a smaller time step produces a more accurate computational result. We decrease the time step until a further decrease of $\Delta t$ gives a difference in chemical speed less than $0.3 \%$. In summary, our simulations are for a $200 \times 15$ mesh with $\Delta z=0.1, \Delta x=0.2$, and $\Delta t=2 \times 10^{-6}$. We also change the mesh size in the $x$ direction to get slabs of different widths.

We run simulations for fronts propagating in slabs of different widths. The initial conditions consist of no fluid flow anywhere. The initial chemical-concentration profiles are set to the convectionless reaction-diffusion pulse plus some small random perturbations in the vicinity of the front. The initial perturbation dies out if the system is below the onset of convection. The fluid velocity gradually increases if the system is above the onset of convection and eventually reaches a steady value. The convective flow travels with the chemical wave as it propagates along the slab. Since we are using a finite-size mesh, the wave will eventually reach the end of the mesh. We can use a larger mesh to allow for longer evolution or solve the system in a comoving frame. But after some test runs, we instead decide to shift the whole system back whenever the pulse is close to the end. The way we determine at what point we should shift the pulse back is to allow the wave to propagate as close to the end as possible, while maintaining that there is no observable difference after shifting. This method has been compared to the two other methods and is computationally more efficient.

\section{RESULTS}

\section{A. Ascending waves in a vertical slab}

We investigate the onset of convection for both vertical and horizontal slabs with a lighter reacting fluid $(\beta>0)$. For ascending waves in a vertical slab with the slab width less than or equal to $0.35 \mathrm{~mm}$, chemical waves propagate upward with the constant speed $C=17.41$ and without any front deformation. In this case, any initial perturbation eventually decays to zero. If the slab width is greater than or equal to $0.37 \mathrm{~mm}$, the initial perturbation gradually evolves into two counterrotating rolls propagating with the chemical wave in the vicinity of the front and the tail, respectively [Fig. 2(b)]. Figure 3 shows the convective fluid velocity field and the shape of the chemical-wave front and the tail. The underlying fluid flow raises the wave front on one side of the slab and lowers it on the opposite side. The wave form and the velocity field are not purely antisymmetric, because the solution includes contributions from the even functions $C_{1}(x), C_{2}(x)$ as well as the odd functions $S_{1}(x), S_{2}(x)$. Above the onset of convection, the chemical-wave velocity increases almost linearly with the slab width (Fig. 4).

To understand the form of the wave front and the direction of the rolls, we refer to the eikonal relation for curved chemical-wave propagation $C=C_{0}-D \kappa$, where $C$ is the normal velocity of a curved chemical wave, $C_{0}$ is the wave velocity of a flat front, $D$ is the molecular diffusivity, and $\kappa$ is the curvature of the front. This relation, an asymptotic form of the reaction-diffusion equations for wave propagations, has been successfully applied to spiral waves in the $\mathrm{BZ}$ reaction [10] as well as to convective chemical waves in the iodate-arsenous acid systems $[8,14]$. In our case (Fig. 3 ), the eikonal relation tends to flatten the front by increasing the velocity on the left side $(\kappa<0)$ and reducing the velocity on the right side $(\kappa>0)$. Without convection, the curved front would not be stable and would eventually evolve into a flat front. Fluid motion balances the effect of the eikonal re- 


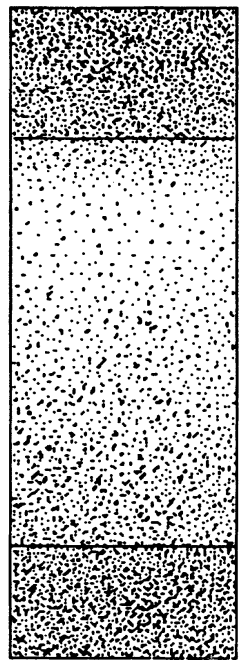

(a)

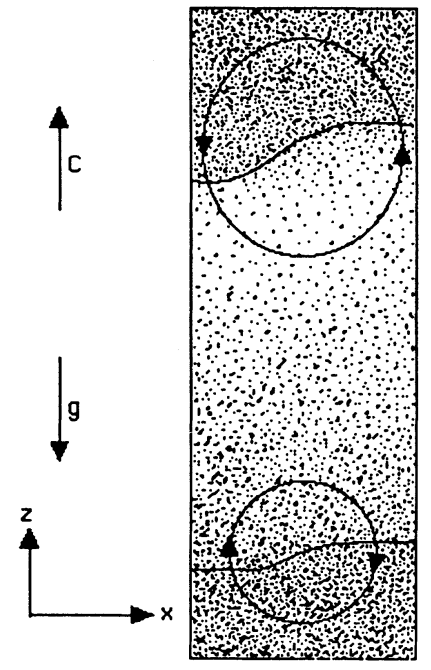

(b)
FIG. 2. Schematic representation of the density profile induced by a chemical wave propagation in the $\mathrm{BZ}$ reaction. For upward propagation (a) the front is unstable, while for downward propagation the tail is unstable. Above the onset of convection (b), two counterrotating rolls are formed near the front and the tail. The front curves and the chemical wave velocity increases.

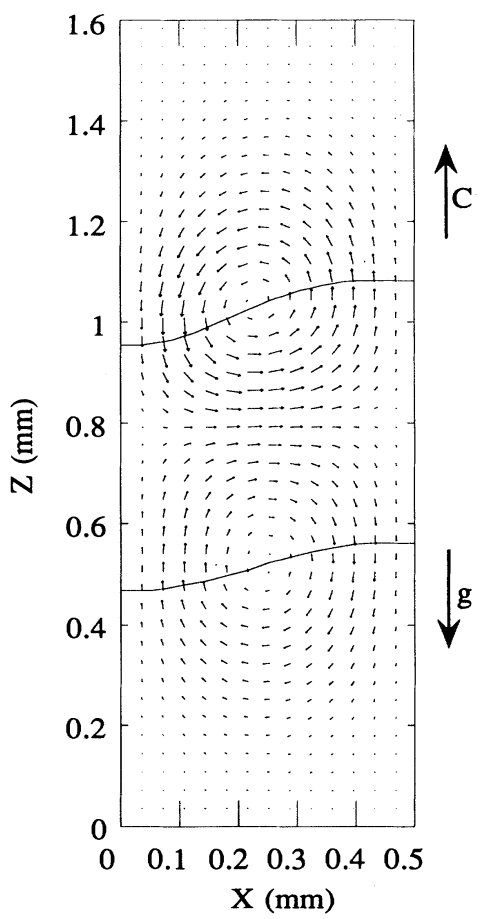

FIG. 3. An ascending wave in a vertical slab with $d=0.50$ $\mathrm{mm}$. The chemical wave is propagating upward with the speed of $C=18.27(9.40 \mathrm{~mm} / \mathrm{min})$. The largest fluid velocities in the upper roll and in the lower roll are 1.03 and $0.69 \mathrm{~mm} / \mathrm{min}$, respectively. The velocity field and waveform are not exactly antiaxisymmetric. The two curves represent the shapes of the front and the tail, respectively.

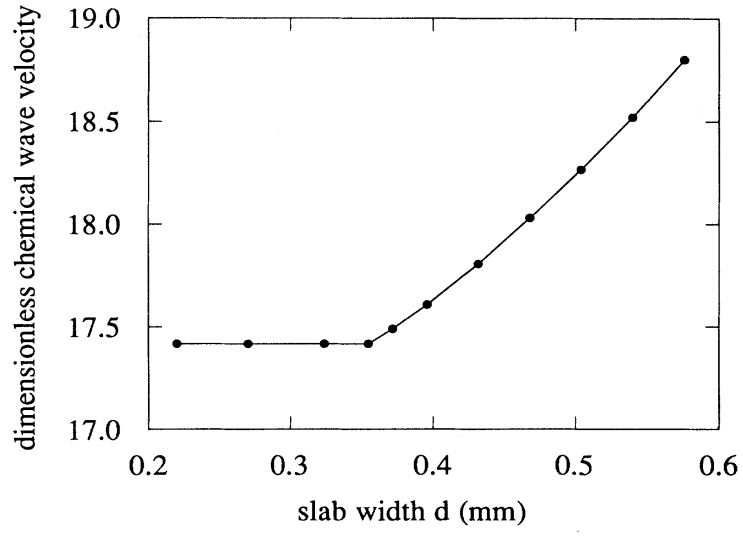

FIG. 4. Chemical wave velocity as a function of slab width for a vertical slab. For slab widths smaller than $0.35 \mathrm{~mm}$, the chemical waves propagate upward with the same velocity as the reaction-diffusion pulse. For slab widths greater than $0.37 \mathrm{~mm}$, the convection is on and the chemical waves travel with speed greater than the reaction-diffusion waves.

lation and allows the curved front to propagate steadily. This explains the physical mechanism for the formation of the first roll near the front. To explain the second roll near the tail, we note that the density gradient near the tail is gravitationally stable and there should be no fluid flow near the tail [Fig. 2(a)]. But the front and the tail are parts of an integral pulse solution of the reactiondiffusion equations, so if the front curves, the tail must correspondingly curve. The curved tail produces a gradient in the horizontal direction, which always leads to convection. The direction of motion of the tail roll opposes the imposed tail curvature. Hence we may say that the formation of the first roll and the deformation of the front are caused by the unstable density gradient near the front, while the less vigorous fluid motion near the tail results from the curved tail enforced by the curved front. The upper roll drives the passive lower roll. The fact that the upper roll is stronger and more extended while the lower roll is weaker and narrower supports our argument. We also observe that the front has a higher curvature than the tail, which is consistent with our reasoning.

Above the onset of convection, we observe an almost linear increase of the chemical-wave velocity as indicated by Fig. 4 . We can explain this behavior qualitatively. For the reaction-diffusion equations, it has been shown that molecular diffusion is the main factor limiting the speed of the chemical waves besides kinetics parameters and initial concentrations [1]. In fact, it can be proven $[1,10]$ that the velocity of convectionless pulse is proportional to the square root of the molecular diffusivity $v \propto \sqrt{D}$. The macroscopic fluid motion helps the mixing of the chemical reagents by bringing the unreacted fluid down and the reacted fluid up near the front of the chemical waves, thereby increasing the speed of the chemical waves by effectively enhancing the macroscopic mass transport of chemical reagents [4]. So far, the only experiment report on BZ waves in vertical tubes did not measure the dependence of the wave velocity on the tube di- 
ameter [7], making it impossible to compare with our results. Their large tube size $(4.3 \mathrm{~mm})$ renders their systems far above the onset of convection and may account for their observation of turbulence instead of steady convective propagation [7].

It may be worthwhile to mention an interesting phenomenon observed in our simulations near the critical width. As we increase the slab width through the critical width, the time required for decay of transients in the fluid velocity field increases rapidly as we approach the critical width. After passing the critical point, this time decreases with increasing slab width (Fig. 5). This is what is known as critical slowing down [17], a clear indication of the existence of a critical point. In fact, for one data point $(d=0.36 \mathrm{~mm})$, we even observe slight initial growth and then, later, decay to zero after a long time. This is due to the sensitivity of a system to perturbations when the system is close to a critical point [17].

\section{B. Descending waves in a vertical slab}

For downward propagation with $\beta>0$, the instability occurs at the tail instead of at the front. This is quite different from the situation of the iodate-arsenous acid system, where the front geometry makes the downward propagation always stable and convectionless $[8,9]$. Because the density gradient near the tail is lower than near

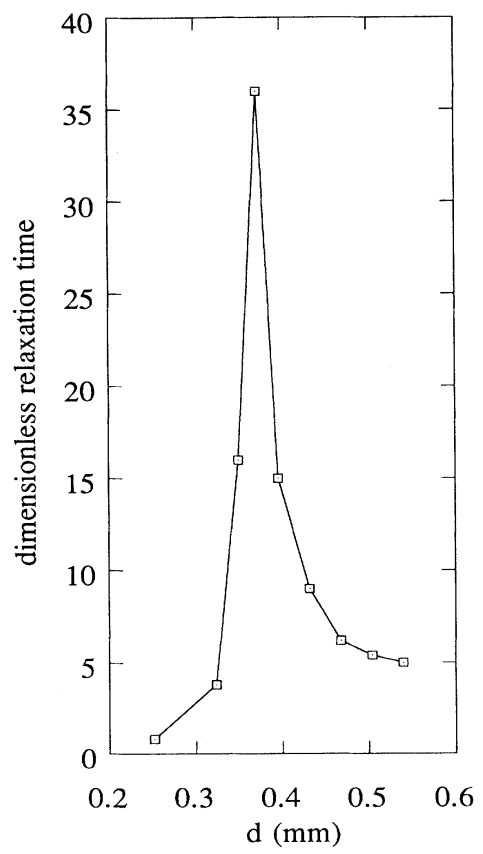

FIG. 5. Relaxation time from initial perturbation to steady wave propagation as a function of slab width. Below the critical width, the relaxation time increases rapidly as we are approaching the critical point. Above the critical width, the relaxation time drops rapidly as the system is taken further above the onset. the front, we expect that the descending waves should have a critical width larger than for the ascending waves. A similar calculation yields a critical width $d_{c}=0.93$ $\mathrm{mm}$, considerably larger than the critical width for ascending waves $\left(d_{c}=0.35 \mathrm{~mm}\right)$. For descending waves above the onset of convection, we observe only one convective roll near the tail instead of two counterrotating rolls (Fig. 6). The tail is strongly deformed to a sinelike form while the front is only slightly curved. Consequently, even though the convective flow near the tail is very strong, with the largest fluid velocity of $5.50 \mathrm{~mm} / \mathrm{min}$ being comparable to the chemical-wave speed, it travels only slightly faster ( $C=17.46 ; 8.98 \mathrm{~mm} / \mathrm{min}$ ) than a pure reaction-diffusion wave $\left(C_{0}=17.41\right)$. This means that the front of the pulse in the $\mathrm{BZ}$ reaction determines the chemical-wave speed. No matter how the tail curves or how strong the flow behind the front is, if the front is not greatly deformed, the pulse will still travel with the reaction-diffusion speed. In this case, we cannot determine the presence of convection by observing the change in the chemical-wave speed. This is similar to the case of the iron-nitric acid reaction, where although convection is observed visually, the convection front travels only slightly faster than the reaction-diffusion front [4]. Even if we increase the slab width to place the system further above the onset of convection, we still observe a single convective roll. But if we tilt the slab slightly (see below), we observe two counter-rotating rolls similar to those in the ascending waves. These results show that the critical

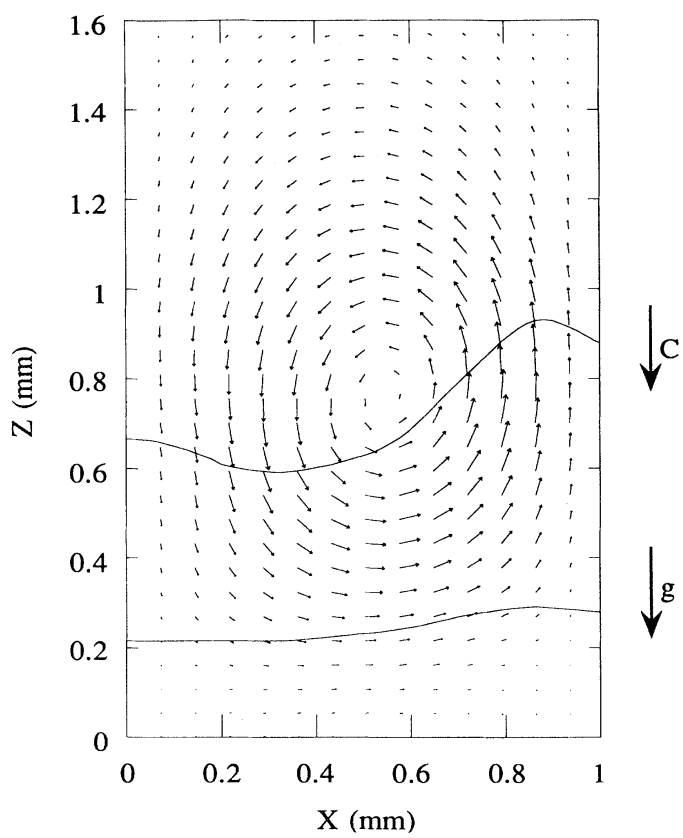

FIG. 6. Fluid velocity field for a descending wave in a vertical slab with $d=1.0 \mathrm{~mm}$. There is only one convective role with the largest fluid velocity $5.50 \mathrm{~mm} / \mathrm{min}$. The tail is strongly curved, but the front is only slightly deformed. The wave travels downward with a speed slightly faster $(C=17.46)$ than the reaction-diffusion wave $\left(C_{0}=17.41\right)$. 
point for the onset of convection is dependent on the density gradients as well as the absolute density change. The ascending and descending waves have the same absolute density change but quite different density gradient, leading to different critical widths.

\section{Horizontal and tilted slabs}

For a horizontal slab, convection always exists because of the existence of the horizontal density gradient [18]. For a small slab width of $0.06 \mathrm{~mm}$, we still observe a steady nonzero velocity field, even though the effect of convection on the chemical-wave speed is negligible. Similar to upward propagation, we observe two counterrotating rolls traveling with the chemical waves [Fig. 7(a)]. The chemical-wave speed increases monotonically with the slab width as shown in Fig. 8. The sharp difference between Figs. 4 and 8 also confirms that there is a critical width for the vertical slab but no critical width for the horizontal slab.

We also carry out a systematic calculation for tilted slabs for three different slab widths: $d_{1}=0.32 \mathrm{~mm}$ (below the onset of convection), $d_{2}=0.47 \mathrm{~mm}$ (above the onset), $d_{3}=0.54 \mathrm{~mm}$ (well above the onset). For any tilted slab, convection is always present. The chemical wave attains a maximum speed at an angle that depends on the width (Fig. 9). We find these angles to be $18.6^{\circ}, 27.3^{\circ}$, and $30.3^{\circ}$ for $d_{1}, d_{2}$, and $d_{3}$ using a least-squares fit. Here we define $\theta=0^{\circ}$ for waves in a horizontal slab and $\theta=90^{\circ}$ for ascending waves in a vertical slab. This effect has been observed experimentally by Nagypal, Bazsa, and Epstein [5] for fronts in the chlorite-thiosulfate reaction and has also been reported in the numerical simulations in the iodate-arsenous acid reaction [9]. A qualitative explanation has been proposed [5]. Since the tilted tube provides a wider cross section for a horizontal plane, convection
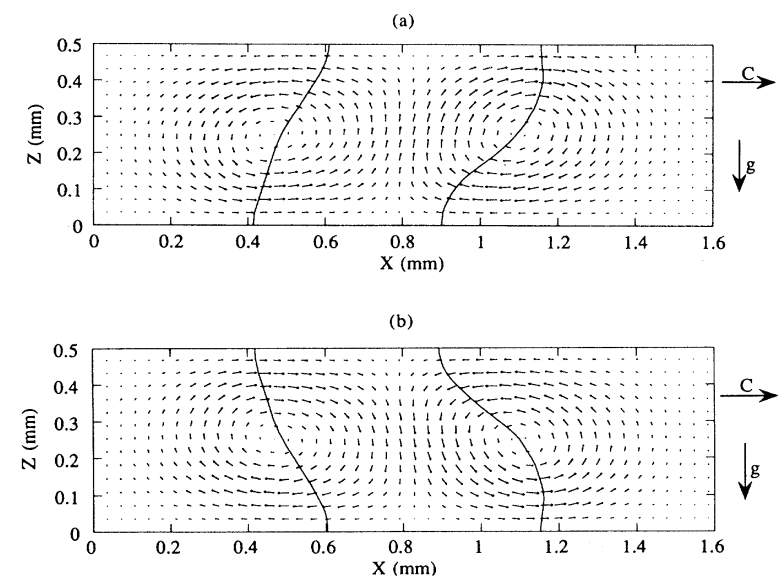

FIG. 7. (a) Fluid velocity field for a horizontal slab with $d=0.50 \mathrm{~mm}$ and $\beta>0$. (b) Fluid velocity field for a horizontal slab with $d=0.50 \mathrm{~mm}$ and $\beta<0$. The chemical wave is traveling to the right at the speed of $10.55 \mathrm{~mm} / \mathrm{min}$ and the largest fluid velocities in the two rolls are 2.60 and $1.98 \mathrm{~mm} / \mathrm{min}$.

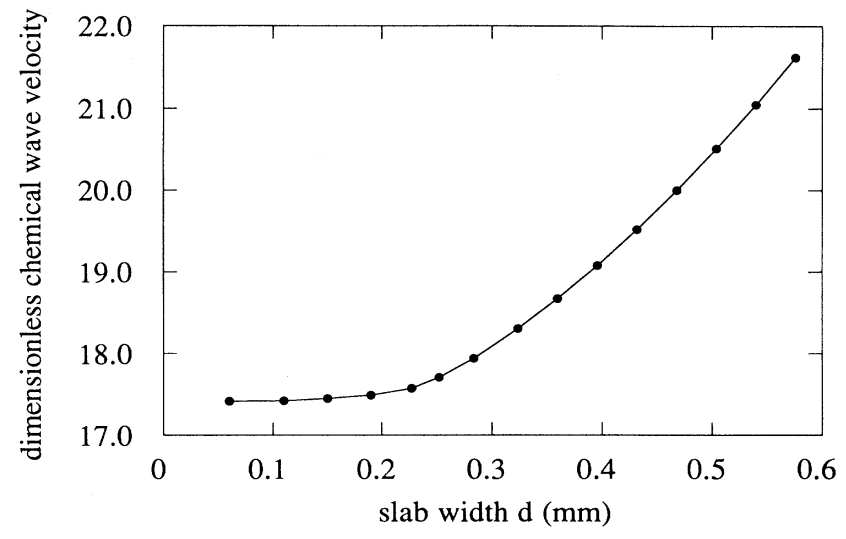

FIG. 8. Chemical wave velocity as a function of the slab width for a horizontal slab. Convection is always present and the chemical waves always travel with speed greater than reaction-diffusion waves.

appears as it does in wider tubes. However, the tilted walls inhibit motion in the vertical direction, effectively slowing the front speed. The competition between these effects provides an angle for maximum speed away from the vertical and horizontal directions.

\section{Heavier reacting fluid $(\beta<0)$}

Up to now, our discussion has been restricted to $\beta>0$. If $\beta<0$ in Eq. (8), the reacting fluid will be heavier than the remaining fluid and the tail will be unstable instead of the front for ascending waves. In fact, the system behavior of Eqs. (10) - (13) is only dependent on the product of $g$ and $\beta$. Reversing the sign of $\beta$ is equivalent to changing the direction of gravity. In other words, for

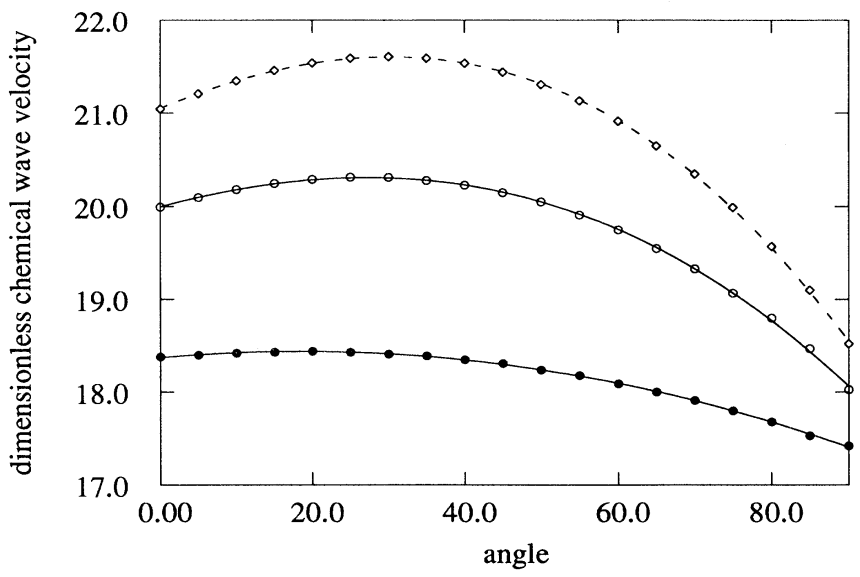

FIG. 9. Chemical wave velocity as a function of the angle for

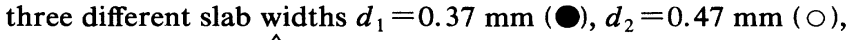
and $d_{3}=0.54 \mathrm{~mm}(\diamond)$. The chemical wave velocities attain the maximum values at $\theta=18.56^{\circ}, 27.30^{\circ}$, and $30.30^{\circ}$ for $d_{1}, d_{2}$, and $d_{3}$. 
$\beta<0$, the results for ascending (descending) waves will be the same as the results for descending (ascending) waves with $\beta>0$.

We can apply the same argument to the horizontal slab. The mirror image of Fig. 7(a) is Fig. 7(b) but with the gravity directing upward. As reversing the sign of both $\beta$ and $g$ simultaneously will not change the system behavior, the system with $\beta<0$ should, and does, have a solution as in Fig. 7(b). If $\beta>0$, we observe the wave form as in Fig. 7(a). If $\beta<0$, we obtain the wave form as in Fig. 7(b). The stability requirement precludes the occurrence of the other wave form. We can see that the heavier fluid moves down and the lighter goes up in both cases. Therefore, two cases with different signs of $\beta$ will have the same dependence of the chemical-wave velocity on slab width as well as the same fluid velocity field. The only difference is the wave shape and the direction of the velocity field. Based on this fact, we can determine the sign of $\beta$, which gives the chemical-composition dependence of the density, just by observing the shape of wave fronts for the $\mathrm{BZ}$ reaction in a petri dish.

Our above results are consistent with the experiments in petri dishes by Miike and co-workers [6] in which they measured the flow velocity distribution by twodimensional spectrophotometry and observed the shape of the wave fronts. In their experiments for the $\mathrm{Fe}^{2+}$. and $\mathrm{Ce}^{3+}$-catalyzed $\mathrm{BZ}$ reaction, Miike and co-workers [6] observe the shape of the wave front to be as in Fig. 7(b) instead of as in Fig. 7(a). In fact, they draw a graph almost identical to Fig. 7(b) in their papers [6]. According to our results, this implies that the reacting fluid is heavier than the remaining fluid $(\beta<0)$ in these experiments. The estimates by Pojman and Epstein [2] for the $\mathrm{Fe}^{2+}$ - and $\mathrm{Ce}^{3+}$-catalyzed $\mathrm{BZ}$ reaction based on the molar volume change also give the same result $(\beta<0)$. In their experiments in the petri dish with a liquid layer thickness $0.85 \mathrm{~mm}$, the chemical wave velocity is about $7.8 \mathrm{~mm} / \mathrm{min}$, and the largest fluid velocities of the first roll and the second roll are $7.5 \mathrm{~mm} / \mathrm{min}$ and 3.0 $\mathrm{mm} / \mathrm{min}$, respectively. In our calculation, the wave velocity for $d=0.50 \mathrm{~mm}$ is $10.55 \mathrm{~mm} / \mathrm{min}$ and the largest velocities of the two rolls are $2.60 \mathrm{~mm} / \mathrm{min}$ and 1.98 $\mathrm{mm} / \mathrm{min}$. Considering the uncertainty in parameter values and widely varying $\beta$, a quantitative comparison is insignificant. Nevertheless, it shows the existence of two rolls with the flow velocity comparable to the velocity of the chemical waves. This result also confirms their assertion that this kind of hydrodynamic flow can be produced exclusively by chemical waves. It also shows that the behavior of the $\mathrm{BZ}$ reaction is catalyst dependent [13]. Our results can be further verified by doing the experiment in a petri dish for the $\mathrm{Mn}^{2+}$-catalyzed $\mathrm{BZ}$ reaction. We predict that the wave front as in Fig. 7(a) should be observed instead of that in Fig. 7(b).

A detailed numerical comparison shows some disagreements between the experiments and our results. The spatial scale of the convective flows in the experiments is much more extended than in our results. Experimentally, the scale of convective flows is about $1 \mathrm{~cm}$, while it is only about $2 \mathrm{~mm}$ in our results. The difference of the largest fluid velocities between the two rolls in the experi- ments is also larger than in our results. We think these disagreements originate from our assumption in Eq. (8). Better numerical agreement with the experimental results may follow by considering the dependence of density on $v$.

\section{CONCLUSION}

The presence of a curved front with traveling convective rolls distinguishes this problem from the classical Rayleigh-Taylor problem. In that problem, two immiscible fluids are placed one on top of the other with surface tension providing the stabilizing mechanism. In our case, the reaction-diffusion mechanism provides a propagating pulse of different density placed between two fluids of the same density. For small tubes, the no-slip boundary condition and the eikonal equation suppress convection. In tubes of larger diameters, the interaction between the stabilizing (reaction-diffusion) and destabilizing (buoyancy) mechanisms leads to the traveling convective rolls. This is not possible in the Rayleigh-Taylor problem because the two fluids are immiscible. This type of convection is also different from Rayleigh-Bénard convection because the convective rolls travel with constant speed and because the instability is driven by a nonlinear pulse profile and not by a linear temperature gradient.

The pulse structure in the $\mathrm{BZ}$ reaction also separates the problem from previous calculations of the front structure in the iodate-arsenous acid system. The most striking difference is that here we have two counterrotating rolls traveling with the chemical waves, while for the front structure in the iodate-arsenous acid system, there is only one convective roll. For the iodate-arsenous acid system, the descending waves are always stable; while for the pulse structure in the $\mathrm{BZ}$ reaction, both ascending and descending waves are unstable (either at the front or at the tail) if the slab width is greater than certain critical widths.

By coupling hydrodynamics with the standard reaction-diffusion equations, we predict an increase of chemical-wave speed as well as a deformation of the front in the $\mathrm{BZ}$ reaction. The increase in speed is due to two counterrotating rolls traveling with the deformed waves. The shape of the front can be explained by the balance between the eikonal relation and the convective flows. For horizontal slabs, we can determine the effect of change in chemical composition on the density by the shape of the front. Two different shapes represent two different density profiles. Our model also shows the existence of an angle for maximum front speed in tilted slabs. Our results agree qualitatively with the existing experiments. We also suggest some simple experiments to test our results. This work shows that convective enhancements are important when comparing reactiondiffusion models with experiments.

Better agreement with the experiments could be achieved by studying the cylindrical geometry. This problem requires treatment in three spatial dimensions, which is considerably more difficult. Currently, we are developing a more accurate code to account for more complicated flows. We intend to consider the depen- 
dence of density on $v$. Many problems remain open for the convective chemical-wave propagation in the $\mathrm{BZ}$ reaction. Among these are convective flows induced by target patterns and spiral waves, the effect of surface evaporative cooling and surface tension, and the occurrence of oscillatory flows.

\section{ACKNOWLEDGMENTS}

This work is supported in part by the National Science Foundation Grant No. RII-8922106 and the National Research Center for Coal and Energy.
[1] R. J. Field and M. Burger, Oscillations and Traveling Waves in Chemical Systems (Wiley, New York, 1985); M. C. Cross and P. C. Hohenberg, Rev. Mod. Phys. 65, 851 (1993).

[2] J. A. Pojman and I. R. Epstein, J. Phys. Chem. 94, 4966 (1990).

[3] T. McManus, Ph.D. thesis, West Virginia University, 1989; J. A. Pojman, I. R. Epstein, T. J. McManus, and K. Showalter, J. Phys. Chem. 95, 1299 (1991); J. Masere, D. A. Vasquez, B. F. Edwards, J. W. Wilder, and K. Showalter, ibid. 98, 6505 (1994).

[4] G. Bazsa and I. R. Epstein, J. Phys. Chem. 89, 3050 (1985); J. A. Pojman and I. R. Epstein, ibid. 95, 1306 (1991).

[5] I. Nagypal, G. Bazsa, and I. R. Epstein, J. Am. Chem. Soc. 108, 3635 (1986).

[6] H. Miike, S. C. Muller, and B. Hess, Chem. Phys. Lett. 144, 515 (1988); Phys. Lett. A 141, 25 (1989); Phys. Rev. Lett. 61, 2109 (1988); H. Miike, H. Yamamoto, S. Kai, and S. C. Muller, Phys. Rev. E 48, 1627 (1993).

[7] M. Menzinger, A. Tzalmona, R. L. Armstrong, A. Cross, and C. Lemaire, J. Phys. Chem. 96, 4725 (1992).

[8] B. F. Edwards, J. W. Wilder, and K. Showalter, Phys. Rev. A 43, 749 (1991); J. W. Wilder, B. F. Edwards, and D. A. Vasquez, Phys. ibid. A 45, 2320 (1991); D. A. Vasquez, B. F. Edwards, and J. W. Wilder (unpublished).
[9] D. A. Vasquez, J. M. Littley, J. W. Wilder, and B. F. Edwards, Phys. Rev. E 50, 280 (1994).

[10] J. P. Keener and J. J. Tyson, Physica D 21, 307 (1986); J. J. Tyson and J. P. Keener, ibid. 32, 327 (1988); J. W. Wilder, D. A. Vasquez, and B. F. Edwards, Phys. Rev. E 47, 3761 (1993).

[11] W. Jahnke, W. E. Skaggs, and A. T. Winfree, J. Phys. Chem. 93, 740 (1989).

[12] T. Plesser, H. Wilke, and K. H. Winters, Chem. Phys. Lett. 200, 158 (1992).

[13] Z. Nagy-Ungvarai, S. C. Muller, Th. Plesser, and B. Hess, Naturwiss. 75, (2), 87 (1988).

[14] D. A. Vasquez, B. F. Edwards, and J. W. Wilder, Phys. Rev. A 43, 6694 (1991); D. A. Vasquez, J. W. Wilder, and B. F. Edwards, Phys. Fluids A 4, 2410 (1992).

[15] S. Chandrasekar, Hydrodynamic and Hydromagnetic Stability (Oxford University Press, London, 1961), Appendix V.

[16] R. Sweet, SIAM (Soc. Ind. Appl. Math.) J. Number. Anal. 14, 706 (1977); J. Adams, P. Swarztrauber, and R. Sweet, FISHPAK, the National Center for Atmospheric Research, Boulder, CO 80307 (1980).

[17] H. E. Stanley, Introduction to Phase Transitions and Critical Phenomena (Oxford University Press, London, 1971).

[18] A. J. Pearlstein, J. Phys. Chem. 89, 1054 (1985). 